\title{
Performance Investigation of a Two Link Manipulator Stability in the Presence of Torque Disturbance using Optimal Sliding Mode Controller
}

\author{
Mustefa Jibril ${ }^{1}$, Messay Tadese ${ }^{2}$, Reta Degefa ${ }^{3}$ \\ ${ }^{1}$ Msc, School of Electrical \& Computer Engineering, Dire Dawa Institute of Technology, Dire Dawa, \\ Ethiopia \\ ${ }^{2}$ Msc, School of Electrical \& Computer Engineering, Dire Dawa Institute of Technology, Dire Dawa, \\ Ethiopia \\ ${ }^{3}$ Msc, School of Electrical \& Computer Engineering, Dire Dawa Institute of Technology, Dire Dawa, \\ Ethiopia \\ mustefa.jibril@ddu.edu.et
}

\begin{abstract}
In this paper, a two-link manipulator system stability performance is designed and analyzed using Optimal control technique. The manipulator system is highly nonlinear and unstable. The system is modelled using Lagrangian equation and linearized in upward unstable position. The closed loop system is designed using optimal sliding mode controller. The system is compared with a known PID controller with an impulse applied and disturbance torques and a promising results has been obtained.
\end{abstract}

Keywords: Manipulator, Sliding mode controller, Proportional integral derivative (PID) controller

\section{Introduction}

In robotics, a manipulator is a system used to manipulate items without any help by the operator. The stubbornness was originally for behavior with radioactive or biohazardous materials, using robotic arms, or they were used in inaccessible places. In more recent development they have been used in diverse pedestal of application including welding automation, robotic surgery and in space. It is an arm-like system that consists of a design of segments, usually sliding or jointed called cross-slides, which nelson and protocol aim with a amounts of degree of freedom. In industrial ergonomics a manipulator is a lift-assist contrivance used to help laborer lift, maneuver and position articles in tendency that are too heavy, too hot, too large or otherwise too difficult for a single worker to manually handle. As opposed to simply vertical lift assists (cranes, hoists, etc.) manipulators have the expertise to sweeps in to tight spaces and remove work pieces. A good form would be banishment large stamped parts from a press and arranging them in a rack or similar dunnage. In welding, a rods boom manipulator is used to reprieve ejection rates, reduce human inaccuracies and other costs in a manufacturing setting. Additionally, manipulator tooling gives the lift assist the aptitude to pitch, roll, or spin the parts for appropriate placement.

\section{System Description}

Figure 1 shows the physical model of a two-link manipulator, with each joint equipped with a motor for providing input torque disturbance, an encoder is used to measure the joint position. The 
objective of the of this system design is to make the joint positions $\theta_{1}$ and $\theta_{2}$ to be stable to the vertical position with the presence of $\mathrm{T} 1$ and $\mathrm{T} 2$ disturbance inputs, which are specified by the vertical system design of the manipulator..

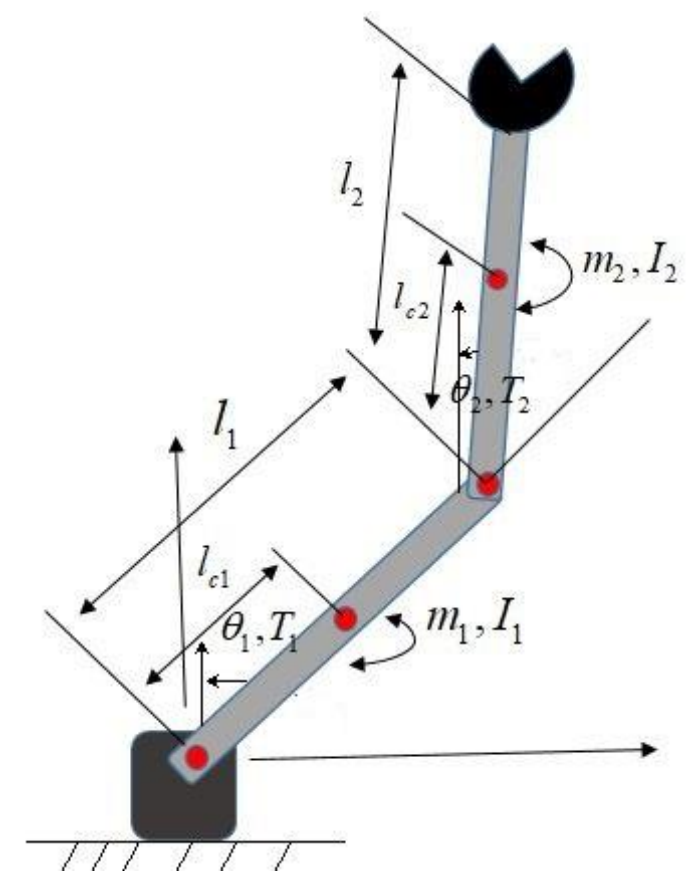

Figure 1 Vertically designed two link manipulator

By using the Lagrangian equations, one can easily show that the dynamic equations of the manipulator are

$$
\left(\begin{array}{ll}
S_{11} & S_{12} \\
S_{21} & S_{22}
\end{array}\right)\left[\begin{array}{l}
\ddot{\theta}_{1} \\
\ddot{\theta}_{2}
\end{array}\right]+\left(\begin{array}{cc}
-M \dot{\theta}_{2} & -M \dot{\theta}_{1}-M \dot{\theta}_{2} \\
M \dot{\theta}_{1} & 0
\end{array}\right)\left[\begin{array}{l}
\dot{\theta}_{1} \\
\dot{\theta}_{2}
\end{array}\right]+\left[\begin{array}{l}
W_{1} \\
W_{2}
\end{array}\right]=\left[\begin{array}{c}
T_{a p p 1}+T_{d i s 1} \\
T_{a p p 2}+T_{d i s 2}
\end{array}\right]
$$

Where

$$
\begin{gathered}
S_{11}=m_{1} l_{C_{1}}{ }^{2}+l_{1}+m_{2}\left(l_{2}^{2}+l_{C_{2}}{ }^{2}+2 l_{2} l_{C_{2}} \cos \theta_{2}\right)+I_{2} \\
S_{22}=m_{2} l_{C_{2}}{ }^{2}+I_{2} \\
S_{12}=S_{21}=m_{2} l_{2} l_{C_{2}} \cos \theta_{2}+m_{2} l_{C_{2}}{ }^{2}+I_{2} \\
M=m_{2} l_{1} l_{C_{2}} \sin \theta_{2} \\
W_{1}=m_{1} l_{C_{1}} g \sin \theta_{1}+m_{2} g \sin \theta_{2}\left(l_{C_{2}} \cos \left(\theta_{1}-\theta_{2}\right)+l_{1} \cos \theta_{1}\right) \\
W_{2}=m_{2} l_{C_{2}} g \sin \left(\theta_{1}-\theta_{2}\right)
\end{gathered}
$$

Where

$T_{a p p 1}$ Torque applied 1 
$T_{a p p 2}$ Torque applied 2

$T_{\text {dis } 1}$ Torque disturbance 1

$T_{\text {dis } 2}$ Torque disturbance 2

\subsection{Linearizing the System}

In this paper, the system linearizing method is done for vertical unstable equilibrium by taking.

$\theta_{1} \approx \theta_{2} \approx 0$

$\cos \theta_{1} \approx \cos \theta_{2} \approx 1$

$\sin \theta_{1} \approx \theta_{1}$

$\sin \theta_{2} \approx \theta_{2}$

$\theta_{1}-\theta_{2} \approx 0$

$\cos \left(\theta_{1}-\theta_{2}\right) \approx 1$

$\sin \left(\theta_{1}-\theta_{2}\right) \approx \theta_{1}-\theta_{2}$

Then Equation (2,3 and 4) becomes

$$
\begin{gathered}
S_{11}=m_{1} l_{C_{1}}{ }^{2}+l_{1}+m_{2}\left(l_{2}{ }^{2}+l_{C_{2}}{ }^{2}+2 l_{2} l_{C_{2}}\right)+I_{2} \\
S_{22}=m_{2} l_{C_{2}}{ }^{2}+I_{2} \\
S_{12}=S_{21}=m_{2} l_{2} l_{C_{2}}+m_{2} l_{C_{2}}{ }^{2}+I_{2} \\
M=m_{2} l_{1} l_{C_{2}} \theta_{2} \\
(6) \\
W_{1}=m_{1} l_{C_{1}} g \theta_{1}+m_{2} g \theta_{2}\left(l_{C_{2}}+l_{1}\right) \\
W_{2}=m_{2} l_{C_{2}} g\left(\theta_{1}-\theta_{2}\right)
\end{gathered}
$$

Then Equation (1) becomes

$$
\begin{aligned}
& \left(\begin{array}{cc}
m_{1} l_{C_{1}}{ }^{2}+l_{1}+m_{2}\left(l_{2}^{2}+l_{C_{2}}{ }^{2}+2 l_{2} l_{C_{2}}\right)+I_{2} & m_{2} l_{2} l_{C_{2}}+m_{2} l_{C_{2}}{ }^{2}+I_{2} \\
m_{2} l_{2} l_{C_{2}}+m_{2} l_{C_{2}}{ }^{2}+I_{2} & m_{2} l_{C_{2}}{ }^{2}+I_{2}
\end{array}\right)\left[\begin{array}{l}
\ddot{\theta}_{1} \\
\ddot{\theta}_{2}
\end{array}\right] \\
& +\left(\begin{array}{cc}
-m_{2} l_{1} l_{C_{2}} & -m_{2} l_{1} l_{C_{2}} \\
0 & 0
\end{array}\right)\left[\begin{array}{l}
\dot{\theta}_{1} \\
\dot{\theta}_{2}
\end{array}\right] \\
& +\left[\begin{array}{ll}
m_{1} l_{C_{1}} g & m_{2} g\left(l_{C_{2}}+l_{1}\right) \\
m_{2} l_{C_{2}} g & -m_{2} l_{C_{2}} g
\end{array}\right]\left[\begin{array}{l}
\theta_{1} \\
\theta_{2}
\end{array}\right]=\left[\begin{array}{l}
T_{1} \\
T_{2}
\end{array}\right]
\end{aligned}
$$

Where 


$$
\begin{gathered}
S=\left(\begin{array}{cc}
m_{1} l_{C_{1}}{ }^{2}+l_{1}+m_{2}\left(l_{2}{ }^{2}+l_{C_{2}}{ }^{2}+2 l_{2} l_{C_{2}}\right)+I_{2} & m_{2} l_{2} l_{C_{2}}+m_{2} l_{C_{2}}{ }^{2}+I_{2} \\
m_{2} l_{2} l_{C_{2}}+m_{2} l_{C_{2}}{ }^{2}+I_{2} & m_{2} l_{C_{2}}{ }^{2}+I_{2}
\end{array}\right) \\
N=\left(\begin{array}{cc}
-m_{2} l_{1} l_{C_{2}} & -m_{2} l_{1} l_{C_{2}} \\
0 & 0
\end{array}\right) \\
W=\left[\begin{array}{cc}
m_{1} l_{C_{1}} g & m_{2} g\left(l_{C_{2}}+l_{1}\right) \\
m_{2} l_{C_{2}} g & -m_{2} l_{C_{2}} g
\end{array}\right] \\
T_{1}=T_{a p p 1}+T_{d i s 1} \\
T_{2}=T_{a p p 2}+T_{d i s 2}
\end{gathered}
$$

The parameters of the system are shown in Table 1 below.

\begin{tabular}{|l|l|l|l|}
\hline No & Parameter & Symbol & Value \\
\hline 1 & Mass of the arm & $m_{1}$ & $4 \mathrm{Kg}$ \\
\hline 2 & Mass of the wrist & $m_{2}$ & $3 \mathrm{Kg}$ \\
\hline 3 & Length of the arm & $l_{1}$ & $0.7 \mathrm{~m}$ \\
\hline 4 & Length of center of mass of arm & $l_{C_{1}}$ & $0.35 \mathrm{~m}$ \\
\hline 5 & Length of the wrist & $l_{2}$ & $0.6 \mathrm{~m}$ \\
\hline 6 & Length of center of mass of wrist & $l_{C_{2}}$ & $0.3 \mathrm{~m}$ \\
\hline 7 & Moment of inertia of arm & $I_{1}$ & $0.8 \mathrm{Kg} \mathrm{m} 2 / \mathrm{s} 2$ \\
\hline 8 & Moment of inertia of wrist & $I_{2}$ & $0.6 \mathrm{Kg} \mathrm{m} 2 / \mathrm{s} 2$ \\
\hline 9 & Gravitational acceleration & $g$ & $10 \mathrm{~m} / \mathrm{s} 2$ \\
\hline
\end{tabular}

The value of the matrix $\mathrm{S}, \mathrm{N}$ and $\mathrm{W}$ becomes

$$
\begin{aligned}
& S=\left(\begin{array}{ll}
4.32 & 1.41 \\
1.41 & 0.87
\end{array}\right) \\
& N=\left(\begin{array}{cc}
-0.63 & -0.63 \\
0 & 0
\end{array}\right) \\
& W=\left[\begin{array}{cc}
12 & 30 \\
9 & -9
\end{array}\right]
\end{aligned}
$$

The system equation becomes

$$
\left(\begin{array}{ll}
4.32 & 1.41 \\
1.41 & 0.87
\end{array}\right)\left[\begin{array}{l}
\ddot{\theta}_{1} \\
\ddot{\theta}_{2}
\end{array}\right]+\left(\begin{array}{cc}
-0.63 & -0.63 \\
0 & 0
\end{array}\right)\left[\begin{array}{l}
\dot{\theta}_{1} \\
\dot{\theta}_{2}
\end{array}\right]+\left[\begin{array}{cc}
12 & 30 \\
9 & -9
\end{array}\right]\left[\begin{array}{l}
\theta_{1} \\
\theta_{2}
\end{array}\right]=\left[\begin{array}{l}
T_{1} \\
T_{2}
\end{array}\right]
$$

Rearranging Equation (9) becomes 


$$
\left[\begin{array}{l}
\ddot{\theta}_{1} \\
\ddot{\theta}_{2}
\end{array}\right]=\left(\begin{array}{cc}
0.3096 & 0.3096 \\
-0.5018 & -0.5018
\end{array}\right)\left[\begin{array}{l}
\dot{\theta}_{1} \\
\dot{\theta}_{2}
\end{array}\right]+\left[\begin{array}{lr}
1.2710 & -21.9115 \\
-12.4047 & 45.8566
\end{array}\right]\left[\begin{array}{l}
\theta_{1} \\
\theta_{2}
\end{array}\right]+\left[\begin{array}{lr}
0.4914 & -0.7965 \\
-0.7965 & 2.4403
\end{array}\right]\left[\begin{array}{l}
T_{1} \\
T_{2}
\end{array}\right]
$$

Let

$$
x_{1}=\theta_{1}, x_{2}=\theta_{2}, x_{3}=\dot{\theta}_{1}, x_{4}=\dot{\theta}_{2}, u_{1}=T_{1} \text { and } u_{2}=T_{2}
$$

So the state space representation of the system becomes

$$
\begin{aligned}
& {\left[\begin{array}{l}
\dot{x}_{1} \\
\dot{x}_{2} \\
\dot{x}_{3} \\
\dot{x}_{4}
\end{array}\right]=\left(\begin{array}{cccc}
0 & 0 & 1 & 0 \\
0 & 0 & 0 & 1 \\
1.2710 & -21.9115 & 0.3096 & 0.3096 \\
-12.4047 & 45.8566 & -0.5018 & -0.5018
\end{array}\right)\left[\begin{array}{l}
x_{1} \\
x_{2} \\
x_{3} \\
x_{4}
\end{array}\right]+\left(\begin{array}{cc}
0 & 0 \\
0 & 0 \\
0.4914 & -0.7965 \\
-0.7965 & 2.4403
\end{array}\right)\left[\begin{array}{l}
u_{1} \\
u_{2}
\end{array}\right]} \\
& y=\left(\begin{array}{llll}
1 & 0 & 0 & 0 \\
0 & 1 & 0 & 0
\end{array}\right)\left[\begin{array}{l}
x_{1} \\
x_{2} \\
x_{3} \\
x_{4}
\end{array}\right]
\end{aligned}
$$

\section{The Proposed Controllers Design}

\subsection{Optimal Sliding Mode Controller}

For the linear system

$$
\dot{x}=A x(t)+B u(t)
$$

the objective is to minimize

$$
J=\int_{0}^{\infty} x^{T} Q x d t
$$

subject to constraints

$$
s(t)=G x(t)=0
$$

Here, the input term is not present in the objective function (16), and the constraints are that the system is on the intersection on $m$ sliding hyperplanes. Furthermore, the matrix $G$ is not specified a priori and will come out as a solution to the problem.

Using the similarity transformation

$$
J=\int_{0}^{\infty} q^{T} Q_{q} q d t
$$

Where 


$$
Q_{q}=\left(H^{-1}\right)^{T} Q H^{-1}
$$

If $Q=Q^{T} \geq 0$, then $Q_{q}=Q_{q}^{T} \geq 0$, then because the signs of eigenvalues are preserved under congruence transformation. Partition to conform to the partition of $\mathbf{q}$ in as follows:

$$
Q_{q}=\left(\begin{array}{cc}
Q_{r} & N \\
N^{T} & R
\end{array}\right)
$$

Then, from (2),

$$
J=\int_{0}^{\infty}\left(q_{1}^{T} Q_{r} q_{1}+2 q_{1}^{T} N q_{2}+q_{2}^{T} R q_{2}\right) d t
$$

When $s(t)=0$, the $(n-m)$ dimensional dynamics is represented by

$$
\dot{q}_{1}=\bar{A}_{11} q_{1}+\bar{A}_{12} q_{2}
$$

Equation 21 and Equation 22 constitute a standard LQ problem provided $R>0$. If $Q$ is chosen to be positive definite, $R$ is guaranteed to be positive definite. In general, $R$ is not guaranteed to be positive definite if $Q$ is positive semidefinite. If $R$ does not turn out to be positive definite, it has to be arbitrarily chosen to be a positive definite matrix. In this case, a new $Q$ will be defined according to (19).

The gain matrix $K$ for the minimum value of $J$ is

$$
K=R^{-1}\left(\bar{A}_{12}^{T} P+N^{T}\right)
$$

Where

$$
\begin{aligned}
& P\left(\bar{A}_{11}-\bar{A}_{12} R^{-1} N^{T}\right)+\left(\bar{A}_{11}{ }^{T}-N R^{-1} \bar{A}_{12}{ }^{T}\right) P-P \bar{A}_{12} R^{-1} \bar{A}_{12}{ }^{T} P+ \\
& Q_{q}-N R^{-1} N^{T}=0
\end{aligned}
$$

For this system the $\mathrm{Q}, \mathrm{R}$ and $\mathrm{N}$ matrices are

$$
Q=\left(\begin{array}{cccc}
1.5 & 0 & 0 & 0 \\
0 & 1.5 & 0 & 0 \\
0 & 0 & 1.5 & 0 \\
0 & 0 & 0 & 1.5
\end{array}\right) R=\left(\begin{array}{cc}
1.25 & 0 \\
0 & 1.25
\end{array}\right) N=\left[\begin{array}{l}
1.32 \\
0.42 \\
1.98 \\
0.12
\end{array}\right]
$$

The gain matrix $\mathrm{K}$ becomes

$$
K=\left[\begin{array}{rrrr}
14.6424 & -25.0976 & 25.3523 & 0.6597 \\
-5.1137 & 47.1712 & -1.1517 & 22.6206
\end{array}\right]
$$




\subsection{PID Controller.}

A proportional-integral-derivative controller (PID) is a mechanism employing feedback that is widely used in industrial control organization and a variety of other implementation requiring continuously modulated control. A PID controller continuously calculates an inaccuracies values as the unlikeness between a desired set point (SP) and a measured process variable (PV) and applies a adjustment based on proportional, integral, and derivative terms (denoted P, I, and D respectively). In practical terms it automatically applies accurate and responsive change to a control function. The controller's PID algorithm restores the measured output to the desired input with minimal deferment and overshoot by increasing the ability of the system. The distinguishing feature of the PID controller is the skill to use the three control terms of proportional, integral and derivative pertinence on the controller output to apply accurate and optimal control.

The proportional, integral, and derivative terms are summed to calculate the output of the PID controller. Defining $\mathrm{u}(\mathrm{t})$ as the controller output, the final term of the PID controller is:

$$
u(t)=K_{p} e(t)+K_{i} \int_{0}^{t} e(\tau) d \tau+K_{d} \frac{d e(t)}{d t}
$$

\subsubsection{Tuning}

The part of these effects is achieved by loop tuning to whip the optimal control function. The tuning constants are denoted as "K" and must be derived for each control application, as they depend on the response wood of the complete loop external to the controller. These are dependent on the behavior of the final control element.

Using Chien, Hrones and Reswick (CHR) PID Tuning Algorithm method the value of the PID controller are

$$
P I D \quad K_{P}=40.4265 \quad K_{I}=97.6709 K_{D}=2.7560
$$

\section{Result and Discussion}

\subsection{Comparison of the Two Link Manipulator with Optimal Sliding Mode and PID Controllers for an Impulse Input Torque 1}

The simulation results of $\theta_{1}$ and $\theta_{2}$ for the comparison of the two link manipulator with optimal sliding mode and PID controllers for an impulse input torque 1 of $0.1 \mathrm{Nm}$ are shown in Figure 2 and Figure 3 respectively. 


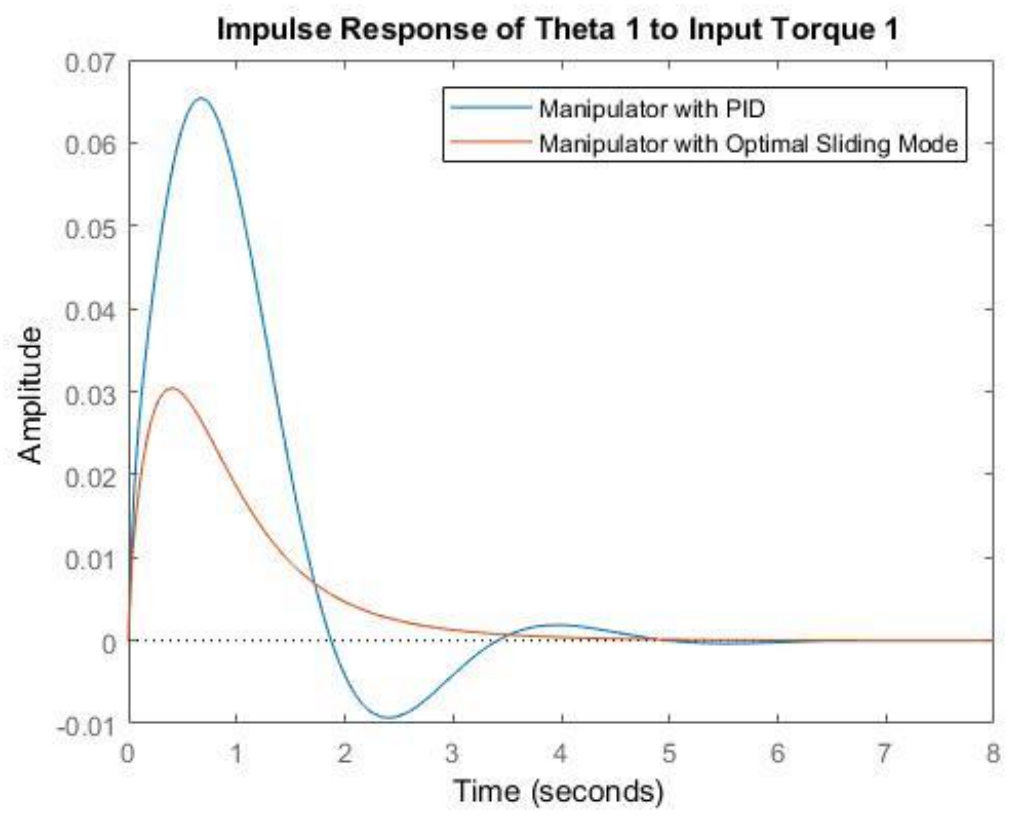

Figure 2 Impulse response of Theta 1 to Torque 1

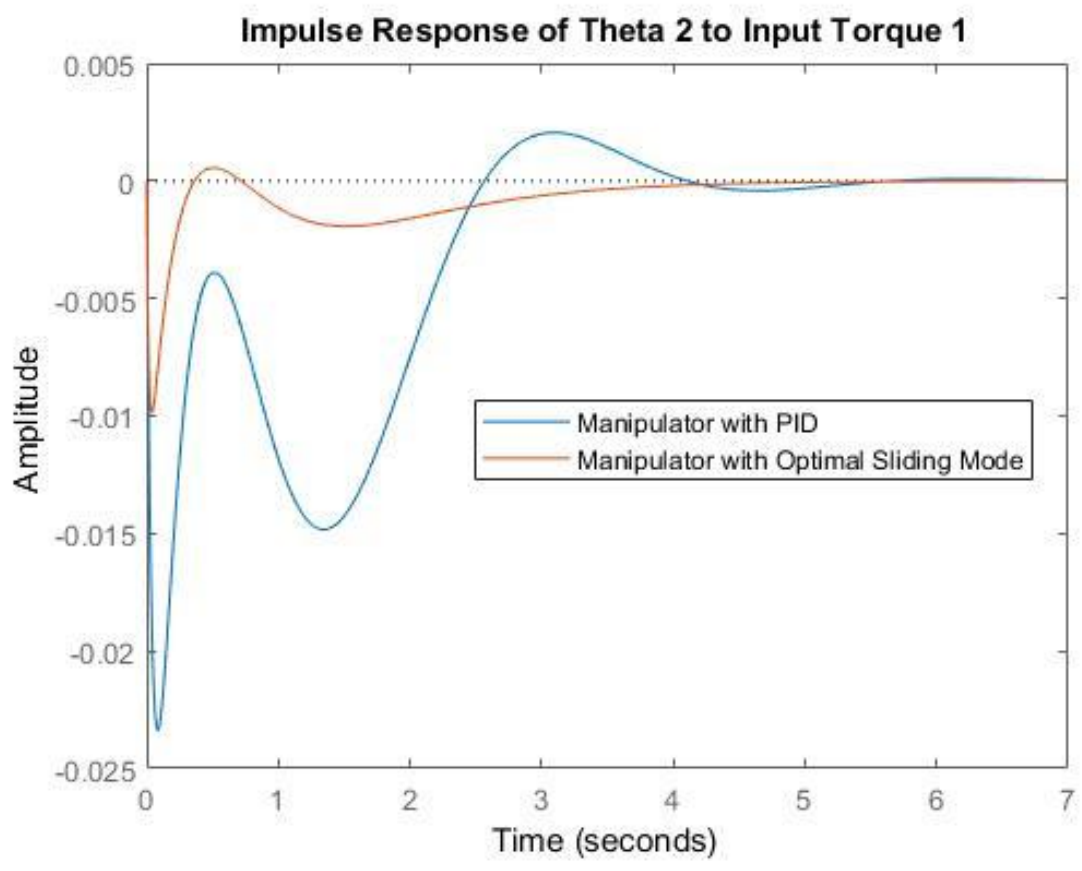

Figure 3 Impulse response of Theta 2 to Torque 1

The simulation result of the impulse response of theta 1 and theta 2 to torque 1 disturbance shows that the manipulator with optimal sliding mode controller minimizes the overshoot and the settling time better than the PID controller. 


\subsection{Comparison of the Two Link Manipulator with Optimal Sliding Mode and PID Controllers for an Impulse Input Torque 2}

The simulation results of $\theta_{1}$ and $\theta_{2}$ for the comparison of the two link manipulator with optimal sliding mode and PID controllers for an impulse input torque 2 of $0.1 \mathrm{Nm}$ are shown in Figure 4 and Figure 5 respectively.

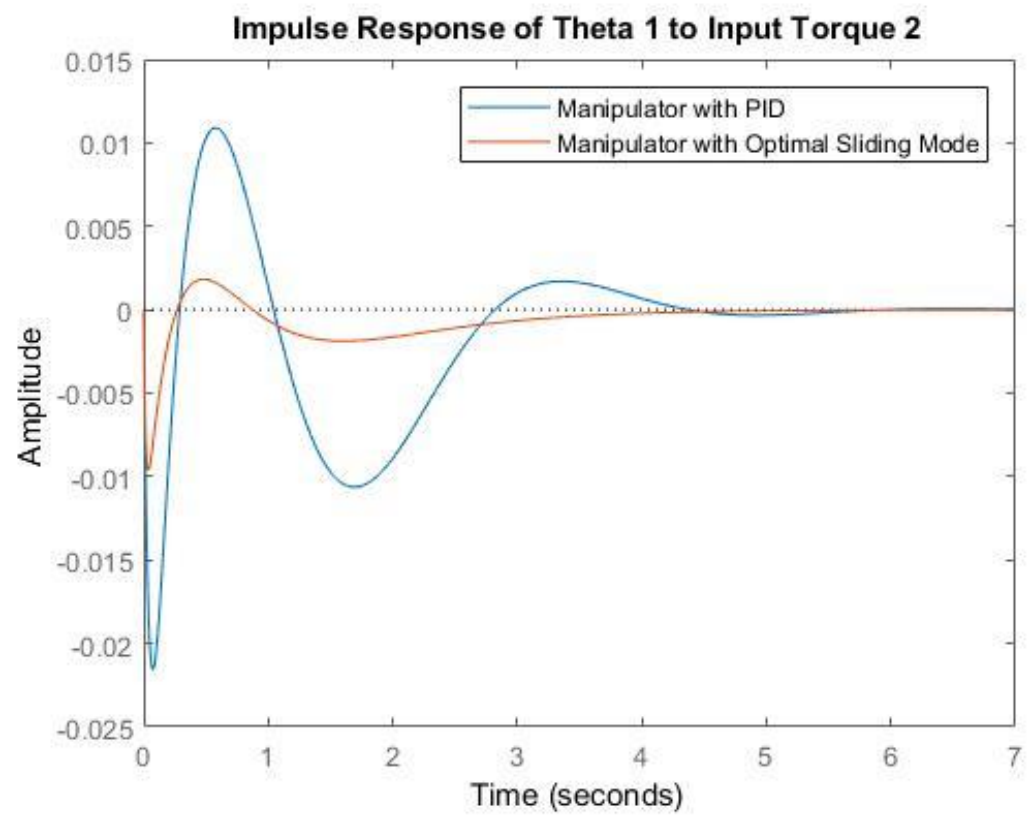

Figure 4 Impulse response of Theta 1 to Torque 2

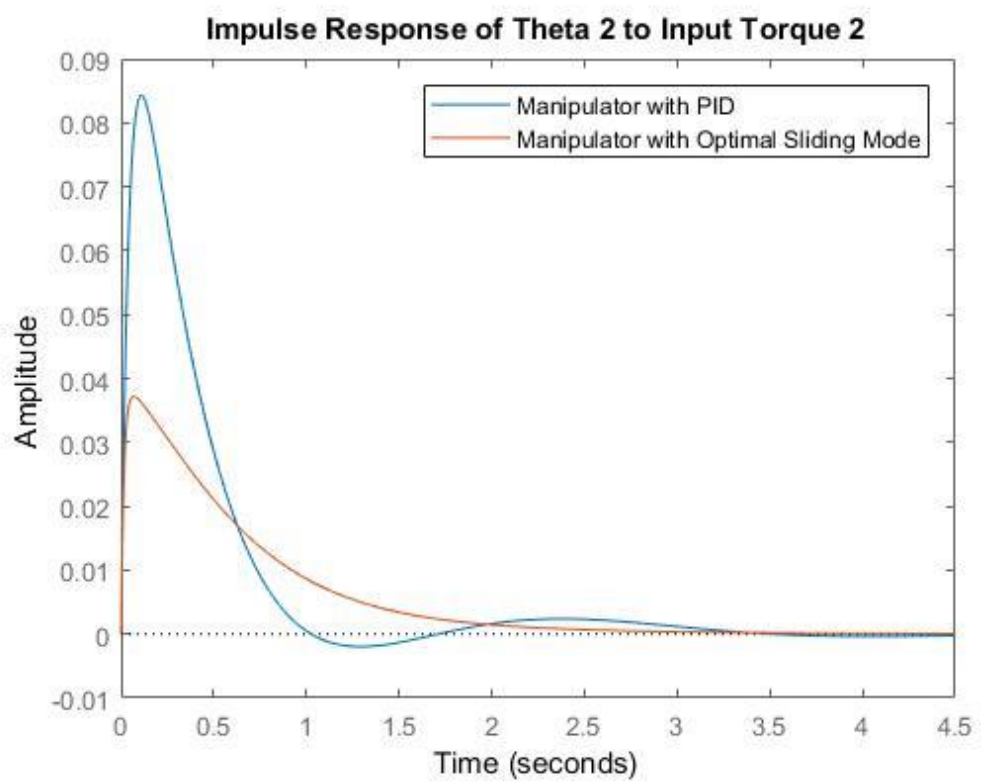

Figure 5 Impulse response of Theta 2 to Torque 2 
The simulation result of the impulse response of theta 1 and theta 2 to torque 2 disturbance shows that the manipulator with optimal sliding mode controller minimizes the overshoot and the settling time better than the PID controller.

\section{Conclusion}

In this paper, stability control of a two link manipulator has been done using optimal sliding mode and proportional integral derivative controllers. The stability performance of the system has been analyzed using comparison simulation between the proposed controllers. The comparison simulation of the two link manipulator with optimal sliding mode and proportional integral derivative controllers has been done for an impulse input of the applied and disturbance torques and the simulation results prove the effectiveness of the proposed optimal sliding mode controller in minimizing the overshoot with a moderate settling time better than the proportional integral derivative controller.

\section{Reference}

[1]. Mustefa Jibril et al. "Position and Speed Control of 2 DOF Industrial Robotic Arm using Robust Controllers" ScienceOpen Preprints, DOI: 10.14293/S2199-1006.1.SOR.PP5YULV.v1, 2020.

[2]. Belkacem R. et al. “Adaptive Neural Network Output Feedback Control for Flexible Multi Link Robotic Manuplators” International Journal of Control, Vol. 92, Issue 10, 2019.

[3].S. Ramalingam et al. "Composite Materials for Advanced Flexible Link Robotic Manipulators: An Investigation” International Journal of Ambient Energy, 2019.

[4]. Xinxin Yang et al. "Dynamic Modelling and Adaptive Robust Tracking Control of a Space Robot with Two Link Flexible Manipulator Under Unknown Disturbances” International Journal of Control, Vol. 91, Issue 4, 2018.

[5].Sun. C. et al. "Fuzzy Neural Network Control of a Flexible Robotic Manipulator using Assumed Mode Method” IEEE Trans. Neural Networks Learn. System, Vol. 29, Issue 11, pp. 5214-5227, 2018.

[6].He. W. et al. "Vibration Control of a Flexible Robotic Manipulator in the presence of Input Dead Zone” IEEE Trans. Ind. Inf., Vol. 13, Issue 1, pp. 48-59, 2017.

[7]. Hroncova D. et al. "A Two Link Manipulator End Effector Control” American Journal of Mechanical Engineering, Vol. 5, Issue 6, pp. 239-242, 2017.

[8]. Alandoli et al. "A Review Study on Flexible Link Manipulators" J. Telecommun. Electron. Comput. Eng. Fig, Vol. 8, Issue 2 pp. 93-97, 2016.

[9]. Mondal S. et al. "Adaptive Second Order Terminal Sliding Mode Controller for Robotic Manipulators” Journal of Franklin Institution, Vol. 351, Issue 4, pp. 2356-2377, 2014. 